\title{
THE ROSAT ALL-SKY SURVEY BRIGHT SOURCE CATALOG
}

W. VOGES, B. ASCHENBACH, Th. BOLLER,

H. BRÄUNINGER, U. BRIEL, W. BURKERT, K. DENNERL,

J. ENGLHAUSER, R. GRUBER, F. HABERL, G. HARTNER,

G. HASINGER, M. KÜRSTER, E. PFEFFERMANN,

W. PIETSCH, P. PREDEHL, C. ROSSO, J.H.M.M. SCHMITT,

J. TRÜMPER AND U. ZIMMERMANN

Max-Planck-Institut für extraterrestrische Physik, Germany

\section{Screening Process}

In order to ensure the quality of the source catalogue derived from the SASS processing an automatic as well as a visual screening procedure was applied to 1378 survey fields. Most (94\%) of the 18,811 sources were confirmed by this screening process. The rest is flagged for various reasons. Broad band images are available for a subset of the flagged sources. Details of the screening process can be found at

www . rosat .mpe-garching.mpg.de/survey/rass-bsc/doc.html.

\section{Catalogue Access}

Lists of the catalogue sources-selected by certain properties-may be retrieved via the ROSAT Source Browser at:

www .rosat .mpe-garching.mpg. de/survey/rass-bsc/scr by .html.

In certain cases, the list provided contains also links to source images. The catalogue can be retrieved via the WWW or anonymous ftp at

(ftp.rosat.mpe-garching.mpg.de; cd archive/survey/rass-bsc). More information on the ROSAT All-Sky Survey Bright Source Catalogue can be found at:

www.rosat .mpe-garching.mpg.de/survey/rass-bsc.html

A list containing correlations with other catalogues and identifications is under preparation and will be available via the World Wide Web. A detailed description of the generation and the scientific content of the ROSAT BSC will be published in Astronomy \& Astrophysics. 


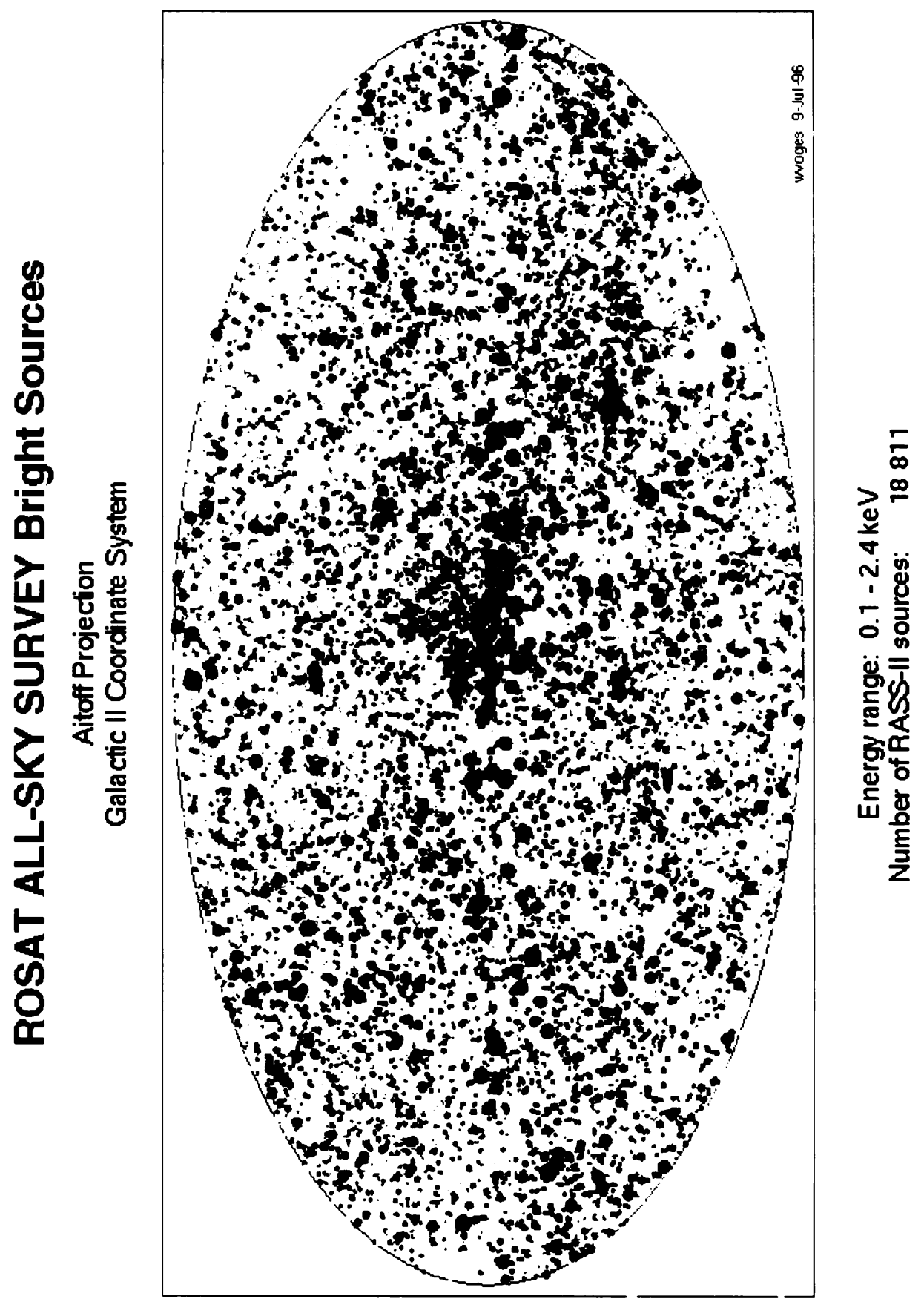

Figure 1. The ROSAT All-Sky Survey Bright Source "atalo ue sources. The originally colour coded hardness ratio is translated in:o a grey , cae light grey: soft a.ld black: hard X-rays). The size of the symbols corres soncis to he source count rate. 